\title{
EL EFECTO DE LA IMPORTACIÓN DE MAQUINARIAS TECNOLÓGICAS EN LAS EXPORTACIONES ECUATORIANAS DE CAMARÓN
}

\section{THE EFFECT OF THE IMPORT OF TECHNOLOGICAL MACHINERY IN ECUADORIAN EXPORTS OF SHRIMP}

\author{
Gabriela Hurtado*, Doménica Zúñiga, Michael Coronel
}

1 Facultad de Ciencias Económicas y Administrativas, Universidad Católica de Santiago de Guayaquil.

\section{RESUMEN}

En el presente trabajo se determinó la relación existente entre la importación en maquinarias tecnológicas con respecto a las exportaciones de camarón en el Ecuador. La investigación de tipo cuantitativo con lógica deductiva, se enfocará en fuentes secundarias del The World Bank y el Banco Central del Ecuador mediante un modelo de Mínimos Cuadrados Ordinarios. Las bases de datos utilizadas fueron analizadas mediante el programa estadístico Stata, en la cual se obtuvo que no existe una relación entre las variables de importación de maquinaria tecnológica y las exportaciones de camarón dado que el valor p no es significante.

Palabras clave: Maquinaria, tecnología, exportaciones de camarón, importación.
In the present work, the relationship between the importation of technological machinery with respect to shrimp exports in Ecuador was determined. The quantitative research with deductive logic, will focus on secondary sources of TheWorld Bank and the Central Bank of Ecuador through an Ordinary Least Squares model. The databases used were analyzed through the Stata statistical program, which showed that there is no relationship between the import variables of technological machinery and exports of shrimp since the $\mathrm{p}$-value is not significant.

Keywords: Machinery, technology, shrimp exports, import. 


\section{Introducción}

A partir del año 2016 se ha registrado una disminución de la importación de equipos de procesamiento de alimentos para el sector camaronero, en consecuencia, con un bajo desempeño de los factores tecnológicos, se asume que, la competitividad de las exportaciones ecuatorianas en el mercado internacional está influenciada en mayor medida por variables tecnológicas en conjunto con el precio internacional establecido para el mercado.

Analizar la relación entre las variables es importante porque la competitividad tecnológica, como estructura y procesos de producción es fundamental para el desempeño exportador, se argumenta por otros autores que las tecnologías influyen en el comercio internacional. Sostienen que los países exportan más porque mediante procesos tecnológicos y estructuras de mercado aumentan las oportunidades y capacidades de exportación de las empresas.

\section{Exportación de camarón en el Ecuador}

Según Chamberlain (2002) la cría de camarón es un negocio que está creciendo rápidamente. En sus primeras etapas, este cultivo confiaba mucho en recursos naturales como las pos-larvas silvestres, los embalses semi-naturales, los alimentos naturales y el uso de grandes ecosistemas colindantes. La cría del camarón es una industria exportadora en Latinoamérica y actualmente hay más de una docena de países con experiencias diversas en este sector, entre los que se destacan: Ecuador, México y Honduras, con alrededor de 180.000, $20.000 \mathrm{y}$ 14.000 hectáreas de estanques, respectivamente. Casi toda la producción de cultivo de estas regiones, está destinada a la exportación, principalmente al mercado de Estados Unidos de América, aunque las dirigidas a las economías europea y japonesa, se están incrementando. Debido a la demanda fuerte y continuada del camarón, al crecimiento de la producción por superficie y al área total de piscinas, esta industria continuará creciendo y puede expandirse a otros países (Tobey, Clay\& Vergne, 1998).

Uzcátegui, Solano y Figueroa (2016) indicaron que la industria camaronera ecuatoriana, con más de cuatro décadas de presencia desde su aparición, muestra un caso exitoso, debido al alto ritmo de crecimiento y convergencia en volúmenes de producción con los principales productores mundiales, como de fracaso, debido al bajo desarrollo de la acción colectiva (capital social) y capacidades tecnológicas propias que le permitieran evitar la tragedia de los comunes que el país sufrió en 1999. En contraparte, los precios internacionales de este commodity atravesó un ciclo expansivo de precios, desde mediados de 2011 hasta mediados de 2015 (International Monetary Found, 2016).

Dentro de este sector agropecuario, Imbs y Wacziarg (2003) señalaron que una mayor diversificación genera, al menos cuando los países son relativamente pobres, ganancias en términos de ingreso per cápita. Contribuyendo a este planteamiento, Torres y Gilles (2013) afirmaron que si existe una alta especialización en pocos productos de exportación, se obtendrá una gran sensibilidad en la economía por las perturbaciones específicas del sector, que generan, a su vez una elevada volatilidad de los ingresos por exportaciones e impactos negativos en el crecimiento.
Bárcena y Prado (2015) observaron que al otro lado del espectro productivo, los países desarrollados son los demandantes de productos básicos y alimentos de manera voraz, y los países emergentes, entre ellos los latinoamericanos, son los proveedores, que hicieron un uso intensivo de los recursos naturales para satisfacer dichas demandas.

\section{Importación de maquinaria tecnológica}

De acuerdo a Tahim, Damaceno y De Araújo Junior (2014)sostuvieronquelaliteraturaneoschumpeteriana destaca las trayectorias tecnológicas que surgen a partir de un paradigma tecnológico que rara vez son impulsadas solo por factores científicos y tecnológicos externos, sino que los factores claves son principalmente económicos y políticos. En este contexto, Tidd, Bessant y Pavitt (2008) sugirieron que la capacidad de las empresas para identificar y explorar sus trayectorias tecnológicas dependerá de la capacidad tecnológica y organizativa específica, así como de la dificultad que los competidores tienen para imitarlos. Esto explica por qué algunas empresas son diferentes y cómo cambian con el tiempo. Dosi (1982) y (2006) definió la trayectoria tecnológica como el conjunto de actividades llevadas a cabo para resolver un problema definido dentro del paradigma tecnológico. En este sentido, el concepto está delimitado por los programas de investigación de tecnología y por el ambiente de selección, que dirigen la respuesta al paradigma tecnológico (Dunham, Bomtempo \& Almeida, 2006).

Natori, et al. (2011) y Rocha (2013)indicaron en sus investigaciones que el cultivo de camarón marino, a pesar de una historia reciente en relación con otros sectores de la acuicultura, constituye el principal vector de desarrollo de tecnologías y servicios para la industria de la acuicultura mundial, favoreciendo su crecimiento rápidamente en muchos países.

\section{El precio internacional del camarón y la exportación de camarón}

De acuerdo a Chamberlain (2002)a medida que la industria fue avanzando, el uso de los elementos del medio ambiente fue disminuyendo progresivamente a favor de un mejor control, eficiencia y sostenibilidad. Al ir aumentando la producción mundial de este crustáceo, los precios comenzaron a decrecer y debido a esto, las granjas camaroneras a nivel internacional luchan por mejorar la eficiencia y reducir los costos de producción. La incertidumbre en la oferta y la demanda están causando fluctuaciones erráticas en los precios, lo que contribuye al desequilibrio del mercado. Según Decamp (2003) el interés por la acuicultura ha estado fundamentado en el cultivo de especies de alto valor comercial como el camarón, que ocupa un lugar cimero a escala mundial comparado con otros renglones, gracias a los convenientes precios en el mercado internacional. La FAO (2009) señala como ejemplo que a pesar del aumento de la demanda, el precio del camarón ha bajado continuamente. Los precios internacionales de este commodity atravesaron un ciclo expansivo de precios, desde mediados de 2011 hasta mediados de 2015 (International Monetary Found, 2016).

Según Hishamunda y Ridler (2002) como uno de los beneficios más importantes que se le pueden atribuir a la actividad acuícola mundial, es digno de mencionar que existe una enorme producción de alimentos de alta calidad a precios accesibles. No 
obstante con el camarón se atribuyen dificultades a nivel mundial por su competencia mundial, según (Neori, et al., 2007) con el camarón se compite por el mercado; en este sentido la sobreoferta de camarón ha abatido los precios

Existen barreras a la exportación, consideradas como limitaciones que impiden a las empresas expandir sus operaciones exportadoras. Entre esas están las barreras Competitivas, conformada por los obstáculos que surgen por la competencia de otras empresas en los mercados extranjeros y la falta de capacidad productiva dedicada a sostener la comercializaciónhacia mercados extranjeros, incluyendo diferentes factores que reflejan la percepción empresarial de lacompetitividad de las empresas en términos de tecnología, servicios postventa, precio y calidad del producto ( (Al-Aali, Jeen$\mathrm{Su}$, \& Al-Aali, 2012); (Leonidou, 200o); (Yannopoulos \& Kefalaki, 2010); (Hutchinson, Fleck, \& Lloyd-Reason, 2009)).

\section{La maquinaria tecnológica y la exportación de camarón}

Valderrama y Neme (2011) sostuvieron como ejemplo el país de México, donde con el mejor desempeño de los factores "tecnológicos" durante el periodo frente a los factores "relacionados con el precio" en un contexto de alto dinamismo exportador de las manufacturas mexicanas, se argumenta que la competitividad de las exportaciones mexicanas en el mercado estadounidense está influenciada en mayor medida por variables tecnológicas. Fagerberg (1996), Krugman (1983) y Grossman y Helpman (1955) argumentaron que la tecnología y diferenciación de producto influyen en el comercio internacional. Los estudios empíricos de la relación entre cambio tecnológico y comercio internacional se dividen en estudios a nivel macroeconómico (Soete, 1987); (Van Hulst, Mulder, \& Soete, 1991); (Cotsomitis, de Bresson , \& Kwan, 1991); (Verspagen \& Wakelin, 1997) y a nivel micro (Greenhalgh, 1990); (Lefebvre, Bourgault, \& Lefebvre, 1998); (Bernard \& Bradford, 2004); (Roper \& Love, 2002); (Love \& Mansury, 2009); (Bleany \& Wakelin, 2002). Las conclusiones en cualquier nivel de análisis son similares al encontrar vínculos positivos entre la actualización tecnológica (empleando diversas variables de tecnología: patentes, actividades de id, etcétera) y el desempeño exportador (aproximado por diferentes indicadores: participación de mercado, probabilidad o decisión de exportar, volumen o valor de las exportaciones).

De acuerdo a Natori, et al. (2011) y Rocha (2006) presentaron que en Brasil, la industria ha sufrido grandes transformaciones desde el año 1978, cuando empieza la producción comercial, hasta el año 1996 con la introducción de un paquete tecnológico para el cultivo de camarón del Pacífico (Litopenaeusvannamei), y más tarde con los avances científicos y tecnológicos en la década de 2000 , donde esta actividad alcanzó su punto máximo en términos de productividad. Esta relación es muy similar en el sector manufacturero, como dijo Álvarez y López (2004), tanto la teoría económica como varios estudios empíricos señalan la existencia de una asociación positiva entre el comercio internacional y la productividad de la industria manufacturera. Según Rozenwurcel y Katz (2012) mantuvieron que las condiciones institucionales y la manera de cada país para lidiar con los desafíos que impone dicho auge son claves para definir las consecuencias del fenómeno (Natori, y otros, 2011).
Método

El presente trabajo cuantitativo mantiene una lógica deductiva, con un alcance correlacional, el cual se centrará en fuentes secundarias del The World Bank (2018) y BCE (2018) con datos de tipo series de tiempo, dado que parte del objetivo planteado y la recolección de información anual de las variables macroeconómicas de importación de maquinaria tecnológica del procesamiento de camarones y exportación de camarón en Ecuador, se enfocará entre los años 1990 y 2017. Además, al ser una investigación no experimental, se implementará un modelo de Mínimos Cuadrados Ordinarios (MCO) para definir si las teorías sustentadas son aplicables a la economía Ecuatoriana y comprobar así las hipótesis planteadas mediante el modelo econométrico, hasta llegar a una conclusión.

Acorde a De los Cobos, Goddard y Gutiérrez (2011) el MCO básicamente consiste en tratar de ajustar un hiperplano que sea compatible con todos los elementos de la muestra, en el que la relación entre Yj y el vector $\mathrm{Xj}$ es lineal $\forall \mathrm{j}$. Sin embargo, hay un elemento de perturbación estocástico. Reconociéndose el término como $\varepsilon j$, que normalmente sigue una distribución normal.

Usualmente se supone que $E[\varepsilon j]=o, \forall j$, la varianza es igual para los errores, $\sigma$, y que éstos están no correlacionados, es decir, $E[\varepsilon \mathrm{k} \varepsilon j]=0, \forall \mathrm{k} 6 \neq \mathrm{j}$, el modelo de estimación de los parámetros es el conocido MCO (De los Cobos, Goddard, \& Gutiérrez, 2011).

Puede escribirse como:

$$
y=X \beta+\varepsilon
$$

Donde según Ramírez y Ramírez (2008)y es un vector de la variable respuesta, $X$ es una matriz $\mathrm{n} \mathrm{x}(\mathrm{p}+1)$ con el intercepto y las $\mathrm{p}$ variables, $\beta$ es el vector de parámetros y $\varepsilon$ es el vector de errores que tiene distribución Normal n-variada con vector de medias o y matriz de varianzas y covarianzas I 2 , con I la matriz identidad nxn.

El estimador de MCO es:

$$
\widehat{\beta}=\left(X^{T} X\right)^{-1} X^{T} Y \text {, con } \operatorname{var}(\widehat{\beta})=\left(X^{T} X\right)^{-1} \sigma^{2}
$$

El modelo de Mínimos Cuadrados Ordinarios permitirá establecer una relación entre la importación de maquinaria tecnológica de procesamiento de camarones y las exportaciones de camarón que sustenta la variación de la variable dependiente, la especificación econométrica de las variables asociadas para la sustentación del índice de confianza del consumidor está dada de la siguiente manera:

Cada una de estos modelos detallados se usará para poder determinar mediante un modelo de regresión la relación que existe entre las importaciones de maquinarias y las exportaciones. El modelo de regresión presentó variables como las que se detallan a continuación.

Variable Dependiente

EXPCAM (Constante) = Nivel de exportación de camarón en el Ecuador (Expresado en dólares) Variables Independientes

IMPMAQ: Nivel de importación de maquinaria para procesamiento de camarón como inversión en infraestructura tecnológica de la industria camaronera (Expresado en dólares) 
PRCCAM: Precio de mercado internacional de camarón (Expresado en dólares)

Entonces, las hipótesis de estudio del presente trabajo investigativo, son las siguientes:

Ho: Las variables de importación de maquinaria tecnológica y el precio internacional del camarón no cumplen un papel sustancial en la determinación del nivel de exportación de camarón en el Ecuador.

H1: Las variables de importación de maquinaria tecnológica y el precio internacional del camarón si cumplen un papel sustancial en la determinación del nivel de exportación de camarón en el Ecuador.

\section{Resultados}

Se especificaron 13 modelos de estimación en la tabla 1, para la cual el modelo con criterios de información AIC y BIC más bajos fue el modelo once, que tiene menos errores y que consiste en evaluar el nivel de exportación de camarón que está en razón de las exportaciones mismas con rezagos de 3 periodos atrás, además de la importación de camarón con rezago de 5 periodos atrás y una diferencia, y el precio internacional de camarón con rezago de 5 periodos atrás y una diferencia.

Tabla 1 Análisis Akaike

\begin{tabular}{lllllll}
\hline Model & Obs & 1l(null) & ll(model) & df & AIC & BIC \\
\hline $\mathrm{dll}$ & 28 & -419.1689 & -408.105 & 3 & 822.2099 & 826.2065 \\
$\mathrm{dl} 2$ & 27 & -404.3849 & -387.6428 & 5 & 785.2856 & 791.7648 \\
$\mathrm{dl} 3$ & 26 & -389.7026 & -366.6152 & 7 & 747.2305 & 756.0371 \\
$\mathrm{dl} 4$ & 25 & -375.0379 & -345.518 & 9 & 709.036 & 720.0059 \\
$\mathrm{dl} 5$ & 24 & -360.3019 & -324.8642 & 11 & 671.7284 & 684.687 \\
$\mathrm{dl6}$ & 23 & -345.6113 & -303.9388 & 13 & 633.877 & 648.6391 \\
$\mathrm{dl} 7$ & 23 & -345.6113 & -303.9388 & 13 & 633.877 & 648.6391 \\
$\mathrm{dl} 8$ & 23 & -345.6113 & -303.9388 & 13 & 633.877 & 648.6391 \\
$\mathrm{dl} 9$ & 23 & -345.6113 & -296.4761 & 14 & 620.9522 & 636.8492 \\
$\mathrm{dll} 10$ & 23 & -345.6113 & -295.0347 & 15 & 620.0693 & 637.1018 \\
$\mathrm{dll1}$ & 23 & -345.6113 & -291.5526 & 16 & 615.1051 & 633.2731 \\
$\mathrm{dll12}$ & 23 & -345.6113 & -291.3391 & 17 & 616.6782 & 635.9816 \\
$\mathrm{dll} 3$ & 23 & -345.6113 & -290.9096 & 18 & 617.8193 & 638.2582 \\
\hline
\end{tabular}

Al observar los resultados de la regresión en la tabla 2, se evidencia la no significancia de las variables pues ningún rezago o diferencia ha influenciado en las exportaciones, a excepción de la importación de maquinaria con rezago de 2 periodos atrás con un nivel de significancia del o,05. Y con un nivel de significancia del o,10 también son significantes las variables de exportación de camarón con rezago de un periodo atrás, y la importación de maquinaria con rezago de 5 periodos atrás.

Tabla 2 Resultados del modelo

\begin{tabular}{|c|c|c|c|c|c|c|}
\hline Expcam & Coef. & $\begin{array}{c}\text { Robust } \\
\text { Std. Err. }\end{array}$ & $\mathrm{t}$ & $P>|t|$ & \multicolumn{2}{|c|}{ (95\% Conf. Interval) } \\
\hline \multicolumn{7}{|l|}{ Expcam } \\
\hline L1. & .6846907 & .3276273 & 2.09 & 0.075 & -.0900249 & 1.459406 \\
\hline L2. & .0048348 & .3959427 & 0.01 & 0.991 & -.9314209 & .9410904 \\
\hline L3. & -.5497743 & .3058307 & -1.80 & 0.115 & -1.272949 & .1734004 \\
\hline \multicolumn{7}{|l|}{ IMPMAQ } \\
\hline.-- & 372.5547 & 122.2907 & 3.05 & 0.019 & 83.38312 & 661.7263 \\
\hline L1. & o & (omitted) & & & & \\
\hline L2. & 231.7729 & 88.54676 & 2.62 & 0.035 & 22.39308 & 441.1527 \\
\hline L3. & 230.8006 & 91.83973 & 2.51 & 0.040 & 13.63417 & 447.9671 \\
\hline L4. & 157.64 & 72.43135 & 2.18 & 0.066 & -13.63295 & 328.9129 \\
\hline L5. & 106.1804 & 47.58252 & 2.23 & 0.061 & -6.334407 & 218.6952 \\
\hline D1. & -157.0798 & 79.45686 & -1.98 & 0.089 & -344.9654 & 30.80579 \\
\hline \multicolumn{7}{|l|}{ PRCCAM } \\
\hline-- & 45763.42 & 24130.41 & 1.90 & 0.100 & -11295.93 & 102822.8 \\
\hline L1. & -51972.96 & 32421.42 & -1.60 & 0.153 & -128637.4 & 24691.53 \\
\hline L2. & -51455.3 & 42190.39 & -1.22 & 0.262 & -151219.7 & 48309.12 \\
\hline L3. & -9967.931 & 35143.34 & -0.28 & 0.785 & -93068.73 & 73132.87 \\
\hline L4. & 45734.38 & 38190.17 & 1.20 & 0.270 & -44571.03 & 136039.8 \\
\hline L5. & -43135.05 & 41126.6 & -1.05 & 0.329 & -140384 & 54113.91 \\
\hline D1. & 0 & (omitted) & & & & \\
\hline _cons & 31577.64 & 271413.9 & 0.12 & 0.911 & -610214.2 & 673369.5 \\
\hline
\end{tabular}


Se pueden interpretar resultados importantes en el modelo registrado en la tabla 2 en donde se puede observar que la variable importación se relaciona con la variable exportación con un nivel de significancia del 5\% para uno y dos rezagos.

La probabilidad Chi2 del modelo es de $32 \%$, la varia- bilidad es estacionaria de manera que un modelo de regresión lineal se considera bueno porque los residuos son White-Noise, aleatorios o estacionarios, distribuidos independientemente, se puede observar la normalidad de los errores para cada modelo especificado en la tabla 3 .

Tabla 3 Prueba de normalidad

\begin{tabular}{lccccc}
\hline & & & & \multicolumn{2}{c}{ - } \\
Variable & Obs & $\operatorname{Pr}$ (Skewness) & $\operatorname{Pr}$ (Kurtosis) & adj chi2 (2) & Prob>chi2 \\
\hline e1 & 28 & 0.1530 & 0.3600 & 3.17 & 0.2045 \\
e2 & 27 & 0.1629 & 0.7202 & 2.28 & 0.3206 \\
e3 & 26 & 0.2329 & 0.6990 & 1.71 & 0.4244 \\
e4 & 25 & 0.3748 & 0.1373 & 3.34 & 0.1884 \\
e5 & 24 & 0.9880 & 0.1629 & 2.15 & 0.3411 \\
e6 & 23 & 0.7369 & 0.9819 & 0.11 & 0.9449 \\
e7 & 23 & 0.7369 & 0.9819 & 0.11 & 0.9449 \\
e8 & 23 & 0.7369 & 0.9819 & 0.11 & 0.9449 \\
e9 & 23 & 0.8948 & 0.6794 & 0.19 & 0.9102 \\
e10 & 23 & 0.4658 & 0.3643 & 1.48 & 0.4767 \\
e11 & 23 & 0.4640 & 0.2248 & 2.23 & 0.3279 \\
e12 & 23 & 0.5680 & 0.2237 & 2.00 & 0.3684 \\
e13 & 23 & 0.8344 & 0.5348 & 0.44 & 0.8016 \\
\hline
\end{tabular}

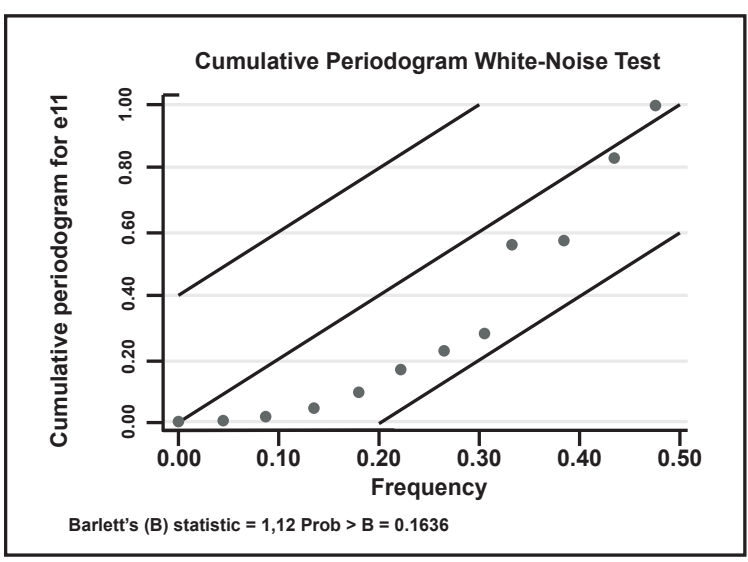

Figura 1. Análisis de Ruido Blanco
A continuación en la figura 1, se desarrolló un análisis de Ruido Blanco para ver si la media y la varianza es constante, se obtuvo el trazado de los residuos y la prueba White-Noise que arrojo una probabilidad de $16 \%$, de manera que el modelo tiene estacionalidad, hay normalidad, hay silencio blanco, la variable residual es aleatoria y estacional.

A continuación, en la tabla 4 se verificó si los residuos son estacionales, considerando que la hipótesis nula del factor Dfuller es raíz unitaria, con un valor $\mathrm{P}$ de o.ooo se rechaza la hipótesis nula, concluyendo que el modelo es estacionario, aplicando los rezagos y diferencias del modelo 11 a partir del año 1990, concluyendo que el modelo es estacionario.

Tabla 4 Test Dickey Fuller

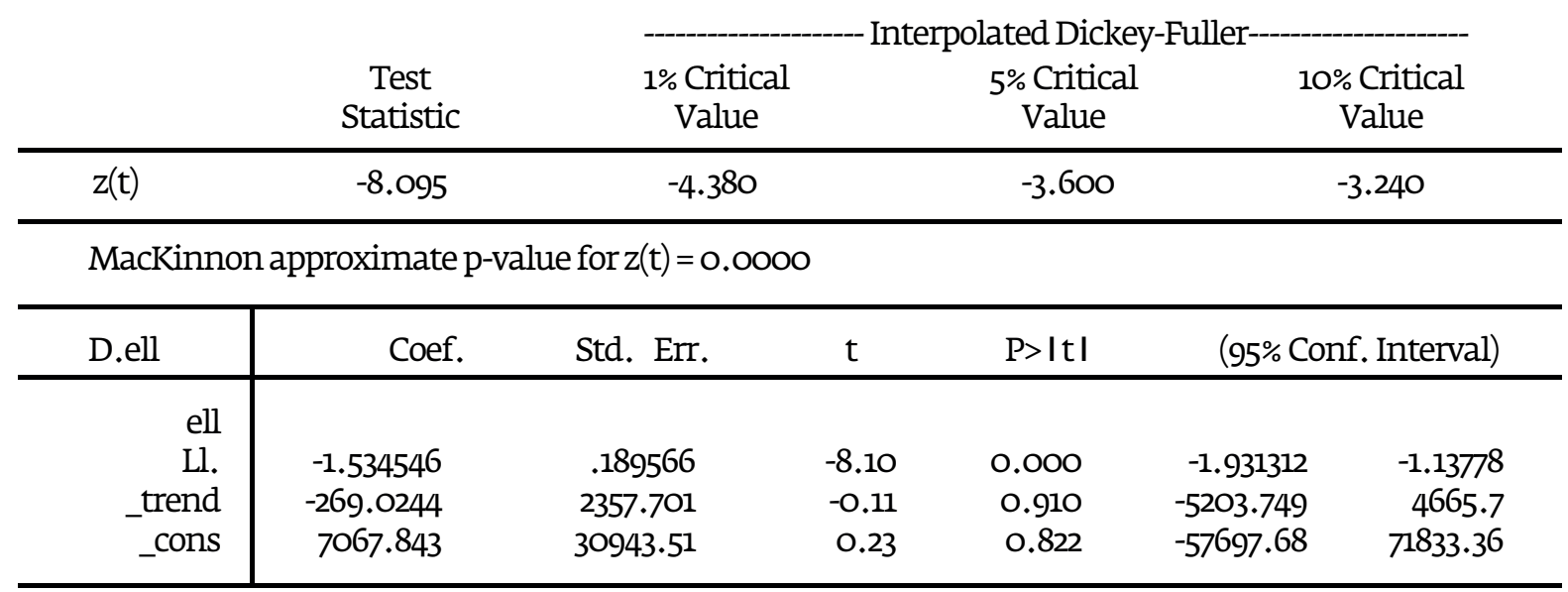




\section{Discusión}

El propósito del estudio es conocer si existe una relación entre la importación de maquinaria tecnológica y la exportación de camarón en el Ecuador. Para ello, se analizaron diferentes investigaciones por otros autores. De acuerdo a un estudio realizado por Valderrama y Neme (2011) indicó que la competitividad de las exportaciones mexicanas en el mercado estadounidense está influenciada en mayor medida por variables tecnológicas, a pesar de una alta variabilidad con respecto al precio en el mercado. Sin embargo, la investigación realizada indicó que no existe una relación entre las variables, puesto que los resultados analizados en el programa estadístico Minitab indicaron que el valor $\mathrm{p}$ de las variables no es significantes considerando un valor beta de o,05.

Por otra parte, Natori, et al. (2011) Indicaron que en Brasil, la industria ha sufrido diversas transformaciones desde el año 1978 hasta el 200o, donde esta actividad pudo lograr lo máximo con respecto a la productividad. Esto quiere decir, que la tecnología influyo en el volumen de exportaciones que lograron alcanzar durante años posteriores a los cambios suscitados. Por otro lado, en la investigación realizada, a pesar de mejorar la tecnología utilizada para poder tratar al camarón y exportarlo a otros países, se ha identificado que no incrementa la participación en mercados internacionales.

\section{Conclusiones}

Como conclusión de la presente investigación, se evidencia que las importaciones de maquinaria en el sector camaronero influyen en los niveles de exportaciones de camarones. La evidencia indica así mismo que el modelo de regresión de las variables importación de maquinaria y precio internacional de camarón no evidencia significancia estadística sobre el nivel de exportaciones de camarón, a excepción de la importación de maquinaria con rezago de dos periodos. Sin embargo, se observa que son significantes las variables de exportación de camarón con rezago de un periodo e importación de maquinaria con rezago de cinco periodos.

No se rechaza la hipótesis de investigación puesto que las variables de importación de maquinaria tecnológica cumplen un papel sustancial en la determinación del nivel de exportación de camarón en el Ecuador. Complementariamente, se constata que el modelo es estacionario y posee normalidad, por otra parte, el precio internacional del camarón no determina una explicación significativa sobre el modelo.

Finalmente, la independencia entre las variables estudiadas indica que la capacidad de la industria camaronera para reconocer que existen otros determinantes para su comportamiento, desarrollo y perspectiva del mercado y su situación económica en la que apuesta su inversión, es función del potencial de la industria camaronera en la economía ecuatoriana.

\section{Referencias}

Al-Aali, A., Jeen-Su, L., \& Al-Aali, H. (2012). Perceived export barrier differences among exporters: Less developed economy evidence. African Journal of Business Management, 6(36), 9945-9956.

Álvarez, R., \& López, R. (2004). Orientación exportadora

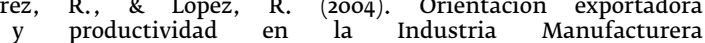

Chilena. Cuadernos de economía, 41(124), 315-343. Bárcena, A., \& Prado, A. (2015). Neoestructuralismo y corrientes heterodoxas en América Latina y el Caribe a inicios del siglo XXI. CEPAL. Obtenido de http://repositorio. cepal.org/bitstream/handle/11362/37648/S1500293_es.pdf BCE. (2018). Banco Central del Ecuador. Obtenido de Comercio Exterior: https://www.bce.fin.ec/index. $\mathrm{php/component/k2/item/35o-comercio-exterior}$ Bernard, A., \& Bradford, J. (2004). Why Some Firms Export. The Review of Economics and Statistics, Vol. 86-2, 561-569.

Bleany, M., \& Wakelin, K. (2002). Efficiency, Innovation and Exports. Oxford Bulletin of Economics and Statistics, Vol, 64-1, 3-15.

Carvalho, S., \& Furtado, A. (2003). Estratégias tecnologicas e dinâmica de inovação das empresas agroalimentares no Brasil. Gestão \& Conexões Management and Connections Journal, 2 (1), 47-75. Chamberlain, G. (2002). Cultivo sostenible de camarón: mitos y realidades. Infofish Internacional 2:11., 23-45. Chamberlain, G. (2002). Cultivo sostenible de camarón: mitos $y$ realidades. Infofish Internacional 2:11. Obtenido de http://www.infopesca.org/articulos/arto6.pdfCotsomitis, J., de Bresson, C., \& Kwan, A. (1991). A Reexamination of the Technology Gap Theory of Trade: Some Evidence from Time Series Data for oecd De los Cobos, S. G., Goddard, J., \& Gutiérrez, M. A. (2011). Regresión borrosa vs. regresión por mínimos cuadrados ordinarios: caso de estudio. Revista de Matematica: Teoría y Aplicaciones 18(1), 33-48.

Decamp, O., Cody, J., Conquest, L., Delanoy, G., \& Tacón, A. (2003). Effect of salinity on natural community and production of Litopenaeus vannamei(Boone) within experimental zerowater exchange culture system. Aquac Research 34., 340-350.

Dosi, G. (1982). Technological Paradigms and Technological Trajectories: A Suggested Interpretation of the Determinants and Directions of Technical Change. Research Policy, 11(3), 147-162.

Dosi, G. (2006). Mudança técnica e transformação industrial: a teoria e uma aplicação à indústria. Campinas: Editora Unicamp..

Dunham, F., Bomtempo, J., \& Almeida, E. (2006). Trajetórias tecnológicas em combustíveis sintéticos: análise dos mecanismos de seleção e indução". Revista Brasileira de Inovação, 5 (1), 99-129.

Fagerberg, J. (1996). Technology and Competitiveness. Oxford Review of Economic, Vol. 12-3, 39-51.

FAO. (30 de Septiembre de 2009). Ayudar a construir un mundo sin de Désitos de documentos de la FAO. http:/ www fanido de Depósitos de documentos de la FAO.. http://www.fao.org/ reenhalgh C. (1990). Innovation and Trade Performance in the United Kingdom. The Economic Journal, Vol. 100, 105-118. Grossman, G., \& Helpman, E. (1955). Technology and trade. R., y P. Kenen (comps.), op. cit., primera edición, Vol. 3, 1279-1337. Hishamunda, N., \& Ridler, N. (2002). Macro policies to promote sustainable commercial aquaculture. Aquacult. Int. 10, 491-505. Hutchinson, K., Fleck, E., \& Lloyd-Reason, L. (2009). An investigation into the initial barriers to internationalization. Evidence from small UK retailers. Journal of Small Business and Enterprise Development, 16 (4), 544-568. Imbs, J., \& Wacziarg, R. (2003). Stages of Diversification. American Economic Review, 93(1), 63-86. International Monetary Found. (2016). International Monetary Found. Obtenido de IMF Primary Commodity Prices: http://www.imf.org/external/np/res/commod/index.aspx Krugman, P. (1983). New Theories of Trade Among Industrial Countries. American Economic Review, Papers and Proceedings, Núm. 73, 343-347. Lefebvre, E., Bourgault, M., \& Lefebvre, L. (1998). r\&d Related Capabilities as Determinants of Export Performance. Journal of Small Business Economics, Vol., 365-377. eonidou, Lours (2000). Barriers to Export Management: an Organizational and Internationalization Analysis. Journal of International Management, 6 (2), 121-148. Love, J., \& Mansury, M. (2009). Exporting and Productivity in Business Services: Evidence from de United States. International Business Review, Vol. 18-2, 630-642.

Morgan, R., \& Katsikeas, C. (1997). Obstacles to Export Initiation and Expansion. . The International Journal of Management, 677-693.

Natori, M., Sussel, F., Santos, E., Previero, T., Viegas, E., \& Gameiro, A. (2011). Desenvolvimento da carcinicultura marinha no Brasil e no mundo: avanços tecnológicos $\mathrm{e}$ desafios. Informações Econômicas, 41 (2), 61-73.

Neori, A Troell, M. Chopin T Yarish, C Critchley, A., \& Buschmann, A. (2007). The need for a balanced ecosystem approach to blue revolution aquaculture. Environment 49, 37-43.

Ramírez, G., \& Ramírez, B. (2008). Colinealidad y mínimos cuadrados ponderados. Revista Venezolana de Análisis de Coyuntura, vol. XII, núm. 1, 283-296.

Rocha, I. (2006). "As perdas de oportunidades pelo setor pesqueiro brasileiro, com ênfase para a carcinicultura marinha: histórico, entraves e perspectivas de recuperação. Revista ABCC, $16(1), 19-23$.

Rocha, J. (2013). A importância da aquicultura e da carcinicultura no contexto da produção mundial de pescado: Desafios e oportunidades para o Brasil",. Revista ABCC, 15 (2), 16-26.

Roper, S., \& Love, J. (2002). Innovation and export performance: evidence from uk and German manufacturing plants. Research Policy, Vol. 31-7,, 1087-1102. Rozenwurcel, G., \& Katz, S. (2012). La economía política de los recursos naturales en América. Revista Integración y Comercio, 35, 19-36.

Silva, C. (2013). . O conceito de Sumak Kawsay e o desenvolvimento equatoriano no governo de Correa: O caso da proposta Yasuní-ITT. Ensaios do IEES, Sao Paulo, 17. 
Soete, L. (1987). The impact of Technological Innovation on International Trade Patterns: The Evidence Reconsidered. Research Policy, Vol. 16-2, 101-130. Tahim, E., Damaceno, M., \& De Araújo Junior, I. (2014). Trayectoria tecnológica einnovación en la industria del cultivo decamarónen el nordeste de Brasil. Revista Galega de Economía, vol. 23, núm, 9-32.

the World Bank. (27 de o8 de 2018). Data Catalog. Obtenido de GEM Commodities: https://datacatalog. world ba n k. org/dat a set/gem-com moditi es

Tidd, J., Bessant, J., \& Pavitt, K. (2008). Gestão da Inovação. $3^{3^{\underline{a}}}$ ed. Porto Alegre: Bookman.

Tigre, P. (2006). Gestão da inovação: a economia da tecnologia no Brasil. Rio de Janeiro: Campus.

Tobey, J., Clay, J., \& Vergne, P. (1998). Manteniendo un Balance: Impactos Económicos, Ambientales y Sociales del Cultivo de Camarón en Latinoamérica. . Reporte de Manejo Costero No 2202. Proyecto de Manejo Costero II. USAID - Centro de Recursos Costeros de la Universidad de Rhode Island., 23-51.
Torres, D. , \& Gilles, E. (2013).TechnologicalStructureofIndustrialExports in Latin America (1990-2010). AD-minister; Número 22, 95-111. Uzcátegui, C., Solano, J., \& Figueroa, P. (2016). Perspectiva sobre la sostenibilidad de los recursos naturales a largo plazo caso industria camaronera ecuatoriana. $\begin{array}{llll}\text { largo plazo caso industria camaronera ecuatoriana. } \\ \text { Revista Universidad y Sociedad 8 } & \text { (3)., 163-168. }\end{array}$ Revista Universidad $\underset{y}{y}$ Sociedad 8 (3)., 163-168.
Valderrama, A., \& Neme, O. (2011). El efecto de la tecnología en las exportaciones manufactureras mexicanas hacia Estados Unidos. Economía: teoría y práctica; Nueva época, número 34, 65-99.

Van Hulst, N., Mulder, R., \& Soete, L. (1991). Exports and Technology in Manufacturing Industry. Review of World Economics, Vol. 127-2, 246-264. Verspagen, B., \& Wakelin, K. (1997). International Competitiveness and its Determinants. International Competitiveness and its Determinants. International
Journal of Applied Economics vol. 11-2, 177-190.

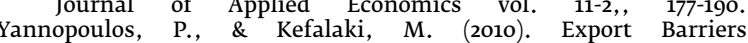

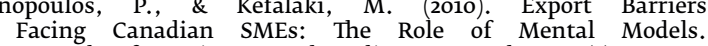
Journal of Business and Policy Research, 5 (2), 54-68. 